\title{
Culturally-based Adaptive Learning and Concept Analytics to Guide Educational Website Content Integration
}

\author{
Torsten Reiners \\ Institute of Information Systems, University of Hamburg, Germany and \\ School of Information Systems, Curtin University of Technology \\ Perth, Western Australia
}

reiners@econ.uni-hamburg.de

Heinz Dreher

School of Information Systems, Curtin University of Technology Perth, Western Australia

\section{h.dreher@curtin.edu.au}

\section{Executive Summary}

In modern learning environments, the lecturer or educational designer is often confronted with multi-national student cohorts, requiring special consideration regarding language, cultural norms and taboos, religion, and ethics. Through a somewhat provocative example we demonstrate that taking such factors into account can be essential to avoid embarrassment and harm to individual learners' cultural sensibilities and, thus, provide the motivation for finding a solution using a specially designed feature, known as adaptive learning paths, for implementation in Learning Management Systems (LMS).

Managing cultural conflicts is achievable by a twofold process. First, a learner profile must be created, in which the specific cultural parameters can be recorded. According to the learner profile, a set of content filter tags can be assigned to the learning path for the relevant students. Example content filter tags may be "no sex" or "nudity ok, but not combined with religion". Second, the LMS must have the functionality to select and present content based on the content filter tags.

The design of learning material is presented via a meta-data based repository of learning objects that permits the adaptation of learning paths according to learner profiles, which include the cultural sensibilities in addition to prior knowledge and learning and categorized learning content $-\mathrm{a}$ detailed example is given.

Material published as part of this publication, either on-line or in print, is copyrighted by the Informing Science Institute. Permission to make digital or paper copy of part or all of these works for personal or classroom use is granted without fee provided that the copies are not made or distributed for profit or commercial advantage AND that copies 1) bear this notice in full and 2) give the full citation on the first page. It is permissible to abstract these works so long as credit is given. To copy in all other cases or to republish or to post on a server or to redistribute to lists requires specific permission and payment of a fee. Contact Publisher@InformingScience.org to request redistribution permission.
The drawback of using static or predefined meta-data elements is discussed, suggesting a further refinement via the introduction of dynamic concept analysis to be applied to both learner profiles and learning objects (restricted to text at this stage). An automated method of generating the content filter tags is achieved through the use of the Normalised Word Vector algorithm first developed for Automated Essay Grading sys- 
tem known as MarkIT (R. Williams, 2006). An automated method reduces human effort and ensures consistency.

Sophisticated fine-grained dynamic learning path adaptivity is achieved through a detailed design given in the article, helping ensure that learners from a variety of cultural backgrounds can be treated appropriately and fairly and are not disadvantaged or offended by inappropriate learning content and examples.

Keywords: cross-cultural education, adaptive learning paths, learner profile, dynamic content selection, concept analysis, Normalised Word Vector algorithm.

\section{Introduction}

Many lecturers assume a homogenous group of participating students since, in general, everyone receives the same learning material. This tendency seems to be independent of whether the education is classroom or virtual learning environment based. Unfortunately, this homogeneity is rarely given in our flat world (Friedman, 2006) as students from all over the entire globe can enroll in the same class or study program, resulting in a potpourri of expectations and demands for the learning experience based on the students' perception of their surrounding and background (Bhattacharya \& Jorgensen, 2004).

The term culture refers to the "set of distinctive spiritual, material, intellectual and emotional features of society or a social group" (UNESCO, 2002) and defines "codes of manners, dress, language, religion, rituals, norms of behaviour such as law and morality" (Jary \& Jary, 1991, p. 101; see also Geertz, 1973; Groeschel \& Doherty, 2000). This definition shows a clear barrier in designing learning material. In view of the codes, we have to state that regarding language it is not acceptable to either encode the learning material in all languages or to force a student to learn another language, i.e. in a virtual e-Learning environment where the students are enrolled from various countries. In addition, certain learning material might not be suitable with respect to the cultural background of the learner. Note that barriers could be compared to the movie rating system, where the society of viewers is divided in groups according to age and suitable content. A discussion on further barriers and constraints such as retrieval, format, or quality is given in Reiners, Reiß, and Sassen (2005) and Schüppel (1997). There is considerable research in the field of cross cultural education (Collis \& Remmers, 1997; Hampden-Turner \& Trompenaars, 2000; Parhizgar, 1998), multicultural learning material and technological solutions (Fluck, 2008; Wang, 2007), and discussion of the role of educators with respect to adapting teaching in multicultural contexts (Bhatti, Tubaisahat, \& El-Qawasmeh, 2005; Larreamendy-Joerns \& Leinhardt, 2006). "Multiculturalism is an issue that is central to educators" (Slay, 2002) and needs to be considered with the "core of school curricula" (Stanley \& Brickhouse, 1994). Nevertheless, we see the focus of this paper not in the argumentation about cultural issues - even though this is of great interest to us and the motivation for our concept - but rather in demonstrating the architecture to achieve a better integration of the individual learner in a learning scenario, that is, the learning material selection and delivery. Furthermore, we consider our concept only as one component in an overall learning environment, which requires further pedagogical and technical parts, such as communication, content design, or teaching methodologies (see, for example, Collis, 1999; McLoughlin, 2001; Rogers, Graham, \& Mayes, 2007; Sims \& Stork, 2007; Young, 2008).

In a classroom setting, individualization is generally very limited as students participate at the same time in the same classroom and, therefore, receive the same presentation with the same slides and the same audio. Most universities require that students know the language for the classes in advance as it is not yet possible to integrate live translations. A further difficulty can result from the content chosen for the lecture as certain subjects might not be compliant with the cultural background of the students. On the other hand, the e-Learning scenario allows individu- 
alization of the learning material by adaptation to a given configuration and dynamic information gathered during the learning process by traversing learning objects with given subjects and concepts.

We believe that culture is highly related to language and, therefore, needs to be modeled as part of the e-Learning system. For the classroom scenario, we are limited to one language for the presentation, whereas integrated components like machine translation would allow on-the-fly translation of objects into the used language; with the limitations that the translation might not be perfect or completely correct (Gaspari, 2006). The same applies to the e-Learning scenario. Nevertheless, another aspect is of importance: in the case of searching, currently only material in the language used to specify the search terms is found; we need cross-language searches on meta-data and concepts, so that additional material is found and used by the learner after being (machine) translated. Here, concepts are "abstract ideas or mental symbols, typically associated with a corresponding representation in a language or symbology" (Wikipedia, 2007).

The suggested concept supports the lecturer by dynamic real-time choice of suitable learning material or examples to demonstrate the latest learned objects, based on the predefined and constantly updated learner user-profile. Moreover, the system analyses the content and prevents visualization of objects that are inappropriate to be shown in the classroom or as part of the virtual course. Note that the system goes beyond the selection of alternative objects but adapts the learning path with respect to previously performed choices and visited objects. The concept shown by Reiners and Sassen (2007) is used and extended to allow further properties to influence the learning path adaptivity and therewith guarantee a cultural sensitivity where no one has to be confronted with inappropriate images, sounds, or text components.

This paper continues with two examples of learning scenarios that demonstrate possible cultural conflicts. In a subsequent section we introduce our learning material design for the multi-lingual adaptive learning path and then describe the integration of concept analysis to improve cultural awareness in the next section. The paper concludes with remarks on the ongoing research and thoughts about how to integrate the system and methodology so as to achieve a high acceptance rate.

\section{Examples of Cultural Conflicts}

From a pedagogical perspective, the understanding of the learning material is improved if theory is supported by descriptive examples. The following two scenarios demonstrate that the selection of these examples might be critical in that the content can be offensive to certain (social) groups and result in a negative learning experience.

The first scenario is about marketing and the hypothesis that "sex sells". Figure 1 shows four examples in which the product's nature is non-sexual but advertised by using sexual associations nudity and even nudity in combination with religion and reference to God. These advertisements might be acceptable in some countries and cultures (and we are not intending to spark a discussion about discrimination and the role of women as sex objects), yet others would either forbid or even punish the display of such pictures. In the U.S., sexual associations and the showing of unclothed people is in daily use, whereas (full) nudity in public is not accepted. In accordance with the religion or cultural background of certain countries, the inhibition threshold might be different and even stricter. 


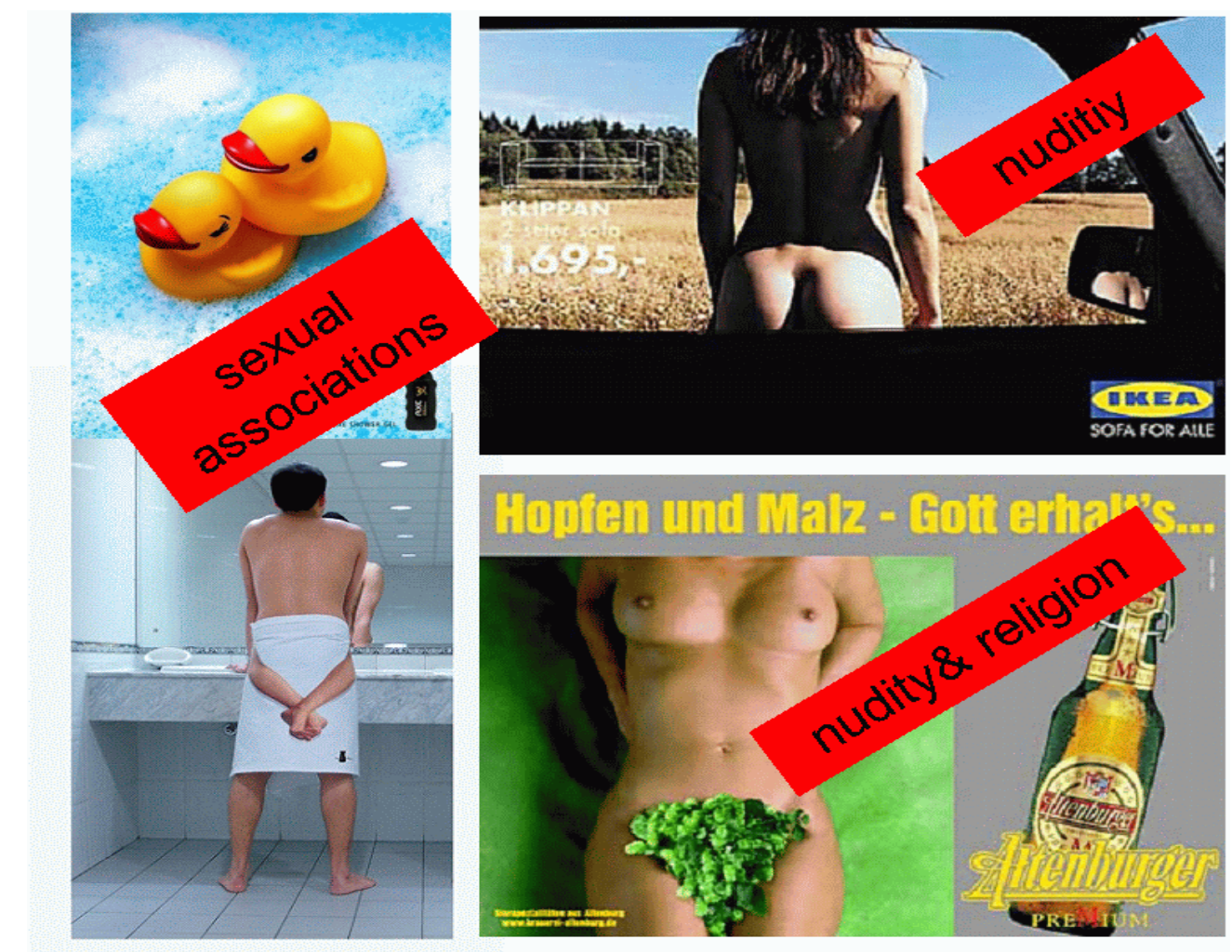

Figure 1: Examples of advertisements with potential conflicts for the students' culture, morality, and ethics. The images might be provocative but demonstrate the major issue of this publication. Those non-adaptive and non-individualized e-learning materials are not appropriate in a multicultural context and require an innovative approach for receiving the right material. Sources of the images are the websites of the companies, printed journals, and websites collecting specialized content and subjects, mainly in the marketing field, and are publicly available.

Another scenario presents the risk of outsourcing production to countries that do not prevent child labor, do not have adequate and safe working conditions, or disregard Intellectual Property Rights (IPR). This could result in a negative impact to the reputation or loss of a company advantage. In the educational lecture setting, scenarios might involve countries of participating students who may be offended by the accusation as their understanding of culture, morality, and ethics is diverse in the interpretation of IPR and cultural norms, for example, "some Chinese companies ... prefer to adopt a 'shortcut' route by launching products based (legally or illegally) on other people's technology rather than investing in the development of their own" (Moynihan et al., 2004), while "taking a more aggressive approach in safeguarding their IPR" (Moynihan et al., 2004; see also Martin, 2007; F. Williams, 2007). Documented cases of similarity in product design are often mentioned in the car industry, for example, using similar logos for car brands, e.g., Toyota vs. Geely Group (Bloomberg, 2003), BMW vs. BYO (F. Williams, 2007) or car designs (see Bradsher, 2007 and Gem Sty, 2007).

Both scenarios point out that the selection of learning material can be crucial for the learning outcome and require support from the learning environment. For the classroom, the examples have to be chosen such that they are appropriate for the majority of students and prevent conflicts with other user groups. In the e-Learning system case, the configuration of the learner profiles allows the dynamic selection of content. That is, for each group the examples for advertisement or outsourcing risk can be selected such that the content is on the border of being allowed or banned and, therefore, is able to be used. For the second scenario, the examples could be selected from 
the perspective of the learner using their country, local culture, and justice system. Note that we could also decide to apply the culture and understanding of morality and ethics of the lecturer, university, or country where the course is offered. Enrolled students from international destinations must typically accept the dominant culture of the host country. Thus, publicly broadcast advertising, for example, whilst possibly offensive to the visitor but not in the dominant culture must be accepted as a condition of the visit (real or virtual). Our designs for learning environments obviate the distracting and distasteful aspects of culture-clashing learning material through the adaptive mechanisms described.

For the implementation, we use individual configurations with elements describing the level of allowed nudity or sexual references and the focus of countries used for scenarios in the examples. Levels could be in the range of none (no nudity or sexual reference) to strong (full nudity and sexually oriented content). For a classroom course, we could set the level according either to the culture of the country - assuming that learners must adapt - or to the minimum level of the participating learners. Note that the first setting would allow a larger flexibility in selecting examples, especially in countries like Germany, where nudity is not generally banned. For the second setting, the selection of learning material is limited to matching examples or needs to be censored using, e.g., cover-patches for areas unsuitable for display.

\section{Design of Learning Material}

The learning material is encoded using hierarchically structured objects of different granularity as shown in Figure 2. Objects are differentiated by their aggregation level in media objects, learning elements, content modules, and thematic meta-structures, whereas the objects themselves are combined by objects of the same or lower level. With respect to reusability, the encoding of the objects should be harmonized and given in as few formats as possible, i.e. concerning the automatic analysis and extraction of information (Reiners \& Sassen, 2007).

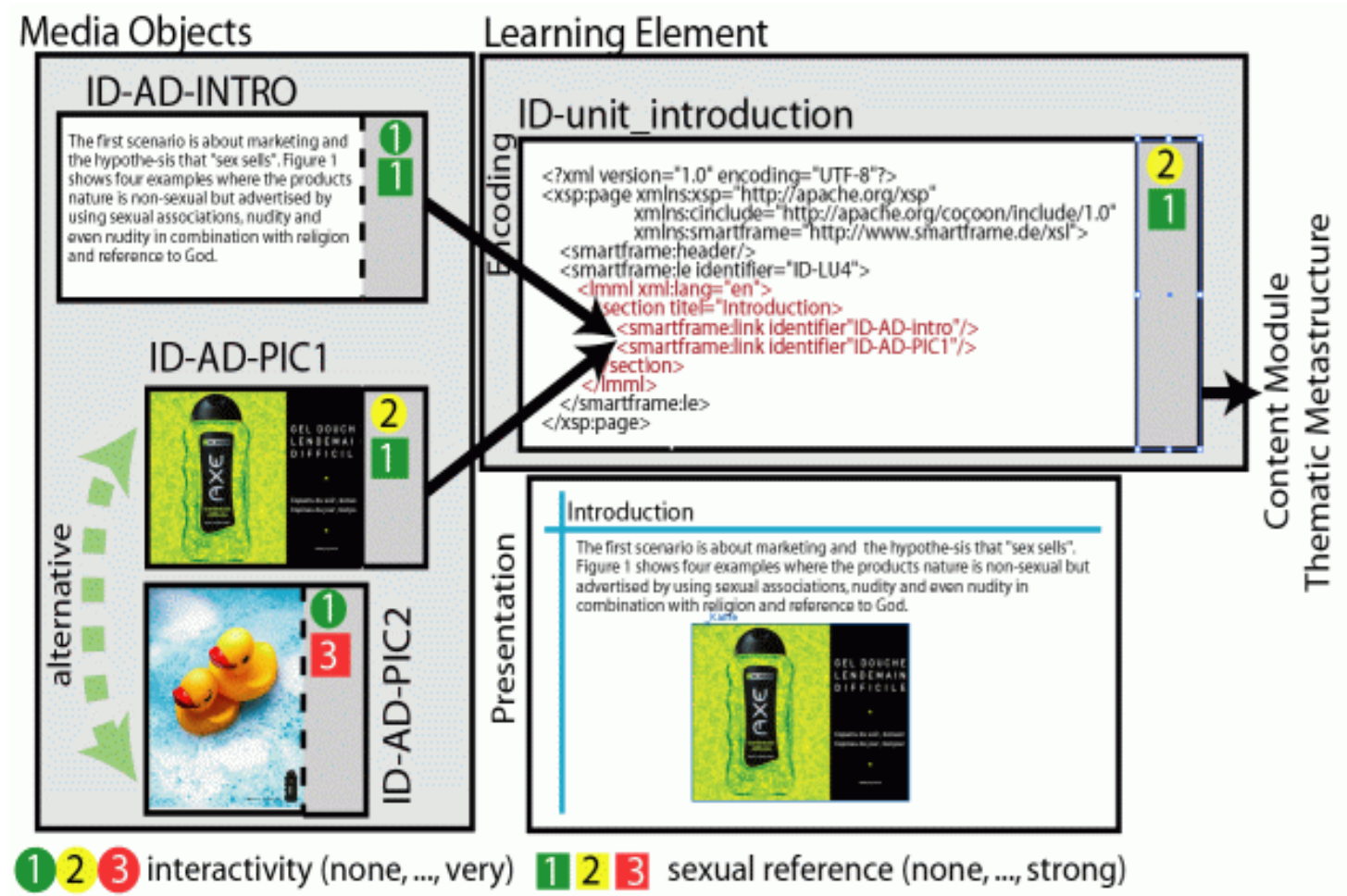

Figure 2: Object-based composition of learning material based on meta-data; based on (Reiners \& Sassen, 2007) 
Connections describe the relation of objects - in Figure 2, the objects ID-AD-PIC1 and ID-ADPIC2 represent alternatives of the same content using, for example, different media, formality, or point of view. Here, we have two advertisements of the same product with different settings for the meta-data as one contains sexual references and is non-interactive while the other one is without sexual references and uses interactive elements to, for example, describe the product. The selection of objects is based on rules and the user configuration by either requiring a strict match or allowing for the best match.

Each object is described by meta-data using a standard like LOM (Learning Object Metadata), Dublin Core (Dublin Core, 2008), or MPEG-7 (Martinez, 2008) with the general understanding of meta-data being still the "authoritative, objective information consisting of facts that do not change" (Nilsson, Palmer, \& Naeve, 2002) describing the properties of the object, from a technical, formal, or educational perspective. This static view is seldom applicable as individuals cannot separate the content from its current context and personal background, configuration, and preferences. For example, Alavi, Maraka, and Yoo (2002) use connotative meta-data to keep "cultural codes that define the culture of a social group" and to prevent misinterpretation and erroneous usage of content objects. Despite the subsequent on-the-fly verification, global and individual tag lists are used to represent the current understanding of content and usage. For example, the user might assign individual tags like night, moon, or scary to an image, whereas another user would rather use the tag romantic instead of scary. The global tags are dynamically generated by an automatic analysis of the objects and the individual tags by, for example, concept analysis using technology such as the Normalised Word Vector (NWV) (R. Williams, 2006) as used in the MarkIT (www.essaygrading.com) Automated Essay Grading system (Dreher, 2006). They can be visualized for the user in so-called tag-clouds representing the importance of certain tags by increased size of the font or as keywords next to visualized objects for further references (HassanMontero \& Herrero-Solana, 2006).

Note that concept analysis or machine translation can also be used to improve the quality of object retrieval as keywords can either be translated to find further content objects in other languages or be expanded using dynamically generated synonyms (see Reiners \& Sassen, 2007, and Dreher \& Williams, 2006, for further details).

\section{Adaptive Learning Paths}

The assumption that the one-size-fits-all method for delivering the learning material in a static manner without any further content and pedagogical revision would only be valid if all individuals learn in the same way (Alavi et al., 2002). Even though the concept of adaptation is discussed in the literature (e.g., Berlanga \& Garcia, 2005; Conlan, Hockemeyer, Wade, \& Albert, 2002; Dreher, Scerbakov, \& Helic, 2004; McAndrew \& Weller, 2005; Sassen \& Schwartz-Reinken, 2005; Towle \& Halm, 2005; Van Rosmalen \& Boticario, 2005), described with concepts (Reiners, Reiß, Schulze, \& Voß, 2003; Reiners \& Sassen, 2007), and demonstrated in first prototypes (Nussbaumer, Gütl, \& Albert, 2007), the methodology to overcome the static and linear learning paths is not yet found in mainstream courseware and virtual learning environments.

Figure 3 describes an example for an adaptive learning path (see especially Reiners \& Sassen, 2007 for a detailed description including further extension of the concept). Here, the first part of an e-learning course "Introduction to Marketing", consisting of four learning objects, is given. Starting with a general object (with the identification term ID-INTRO) for introduction, the learning path continues with a first possible example for marketing campaigns (ID-EXAMPLE) and a basic theoretical description (ID-THEORY). Note that the concept allows several abstraction levels and, therefore, each object might consist of further objects representing full learning units rather than small text blocks or images. The course as well as each object is annotated with metadata describing the content and its properties with respect to educational purposes. Since the fo- 


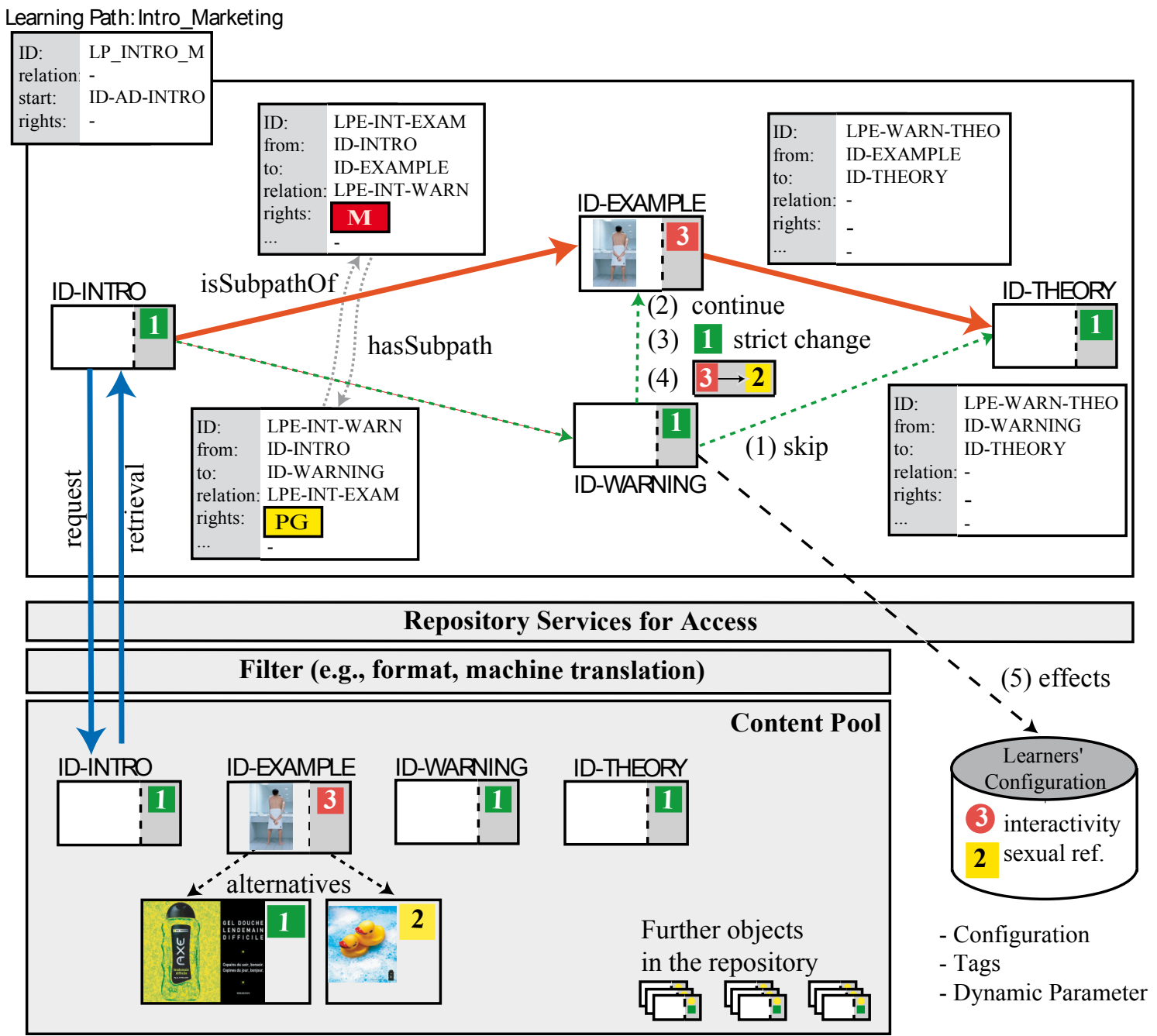

Figure 3: Example of an adaptive learning path (based on Reiners \& Sassen, 2007)

cus is set on the learning path and, therefore, on the relations (edges) between the objects, the only visualized meta-data element is the degree of sexual reference shown by a small colored square on the right side: 1 for non and 3 for strong sexual reference. For the edges the meta-data is reduced to the elements required to describe the scenario of two learners, one being offended by unexpected appearance of nudity or sexual references ( $G$, general audience), while the other is not ( $\mathrm{M}$, mature). The meta-data element rights represents the minimum level that is required by the user in its configuration setting; $G$ for none, $P G$ for mild, and $M$ for strong sexual references. The learning path and edges are annotated with the following elements: unique identification (ID), first object on the learning path (start), rights, the relation to other paths or edges, respectively (relation) and in case of edges the source (from) and target (to) node.

Within the content pool, the objects are interconnected to represent existing relations. That is, the object ID-EXAMPLE with a sexual reference level of 3 is connected to objects representing the same content but using other examples with other levels. Whenever an object is selected for visualization - and adaptivity is allowed - the best match from the set of related objects is chosen.

Note that the selection is based on only one attribute in this example. In case of several attributes, the best match has to be chosen based on the combination of all differences. 
The learning path shown in red - with the objects ID-INTRO, ID-EXAMPLE, ID-THEORY - is the main path defined by the author or lecturer, respectively. Due to the content of the object IDEXAMPLE showing sexual references with a half naked person, the relation between ID-INTRO and ID-EXAMPLE requires a minimum right level $\mathrm{M}$. Other users are not able to follow the intended learning path but are guided to a related path with a lower level of required rights, here edge LPE-INT-WARN from ID-INTRO to ID-WARNING containing a warning message as well as a question how to continue (allowing sexual references, presenting an alternative, or skipping to the next possible object on the learning path). Based on the answer and the configuration of the learner's profile, the following outcomes are possible; see Figure 3 where the item numbers are also given:

1. The user prefers to skip the learning unit ID-EXAMPLE. This (explicit) decision by the learner would result in using edge LPE-WARN-THEO to the learning unit ID-THEORY;

2. Due to the warning, the user knows what to expect and continues with learning object IDEXAMPLE without any substitution;

3. The user wants to see the example but only with content having no sexual references (here marked with a green rectangle with the number 1 . For the next step, the system selects an alternative object for ID-EXAMPLE (which has to be written in advance and stored in the learning object repository). Note that this strict decision would not allow objects with a fixed or best matching distance such as an object having only mild sexual references;

4. The user can set a transformation rule for meta-data elements such that a value " 3 - strong sexual references" is only seen as " 2 - mild sexual references" and, therefore, allowed to be shown. The transformation rule is also applied to other values affecting them in a proportional manner. The learning process continues with element ID-EXAMPLE;

5. The previous cases described temporary changes on the selection process. If the learner allows adaptation to its behavior, the answer - including the effects on his configuration would be stored in the user profile for later usage. Furthermore, the user can explicitly set the element sexual reference in the configuration to strong and continue with the learning path seeing the examples.

In the example in Figure 3, the user's current configuration - shown in the lower right corner would prevent the usage of the main learning path but follow the alternative path. In learning object ID-WARNING, the user might - depending on the user's rights to change the configuration values - choose one of the five possible options described above. Depending on the current mood, the user might decide to allow an object with a higher level than usual and, therefore, would set a temporary transformation rule that would lower all meta-data elements for sexual reference by one level. The user would continue with ID-EXAMPLE on level 3 (strong sexual reference) matching the user's setting of 2 as a result of the variation by one level.

We should point out that the concept for the adaptive learning paths encompasses far more options than shown in the example above, where we had to limit ourselves by simplifying the number of meta-data elements and modeling techniques to focus on how cultural recognition can be realized in different ways. Especially the substitution of objects - based on the previous behavior of the learner - and modification of the content pool like new objects, new relations, or changed meta-data elements are not demonstrated in this contribution and can be read in Reiners and Sas$\operatorname{sen}(2007)$.

Important in this scenario is that the modification of meta-data and configuration elements can be limited for some learners on a specific learning path. Otherwise, a young learner could override mechanisms to prevent him from seeing material that is not appropriate. Furthermore, we distin- 
guish between an adaptation based on the learner's decision (direct or explicit) and behavior without explicitly involving the user (indirect or implicit).

\section{Improving Adaptivity Using Concepts}

The previous section described how the adaptation of the content can be realized using static meta-data elements. This approach has two major drawbacks: (1) the high quantity of required predefined alternatives for each object on the learning path to allow individualization as well as consideration of culture within the selected learning material and (2) the necessity of adequate metadata, tags, and relations to other objects. In addition, it is impossible for the course designer or lecturer to cover all possible scenarios in advance, especially if the system allows reusability of all objects and dynamic composition based on the learning behaviour and configuration of the learner profile. Even though the learning object author is able to define static meta-data for the general or intended usage of the learning object, we need to specify the dynamic view within other contexts or by individuals with different backgrounds. One possible approach is the annotation of objects with tags by users in addition to the meta-data elements, whereas the quality, accuracy, and correctness for matching requests might be decreased or blurred. In particular, without control mechanisms authors can add or omit certain keywords or meta-data elements to pass given barriers and bring objects of inappropriate content into the learning material.

We suggest a dynamic conceptual analysis to define alternatives for objects and, in particular, to prevent the presentation to the wrong audience. Figure 4 shows the object ID-EXAMPLE on the main learning path, which is about displaying an advertisement containing sexual references tagged with the concepts attractive, advertisement, and nudity, whereas the first two are also marked as mandatory. For the course designer and the lecturer, the mandatory concepts are important to guarantee that the relevant subjects are contained in the learning material. The metadata of the learner specify the level 2 regarding the allowed sexual references. Furthermore, the concept set culture 1 - not allowing nudity - as well as the concepts attractive and sexuality are assigned to the learner. The concept sets are stored in a database and represent cultures or other groups. The learner can associate himself with these sets, so that certain concepts would be used for him in the further selection of learning material. Note that learners can add or remove concepts to match their individual characteristics.

Even though the meta-data element sexual reference would match the current object on the learning path, the concept nudity is set for ID-EXAMPLE but not allowed by the user. From the two alternatives (Step 1 in Figure 4) for ID-EXAMPLE, the first one would be allowed from the concepts but not the meta-data and the second one would not match due to the concepts. If the user does not allow any variation in the meta-data or concepts, no object from the predefined alternatives can be displayed.

Next, the repository is searched (Step 2) for an object that matches the concepts attractive and advertisement but not nudity as this is not allowed by the learner's configuration. The objects are either annotated with concepts (tags) during import or on-the-fly during the search process. The best match - an advertisement for PETA having an attractive female dressed like a tiger and, therewith, not showing nudity - is returned in Step 3. The usage of concepts allows a distinct selection of objects. Assume a learner associated with culture2. Objects about animals like pigs are allowed, but if the concept changes, for example, to a supermarket having pork on display, the object would not be suitable. The system would prevent the inclusion of the object in the learning path including the originally planned object as well its alternatives. 


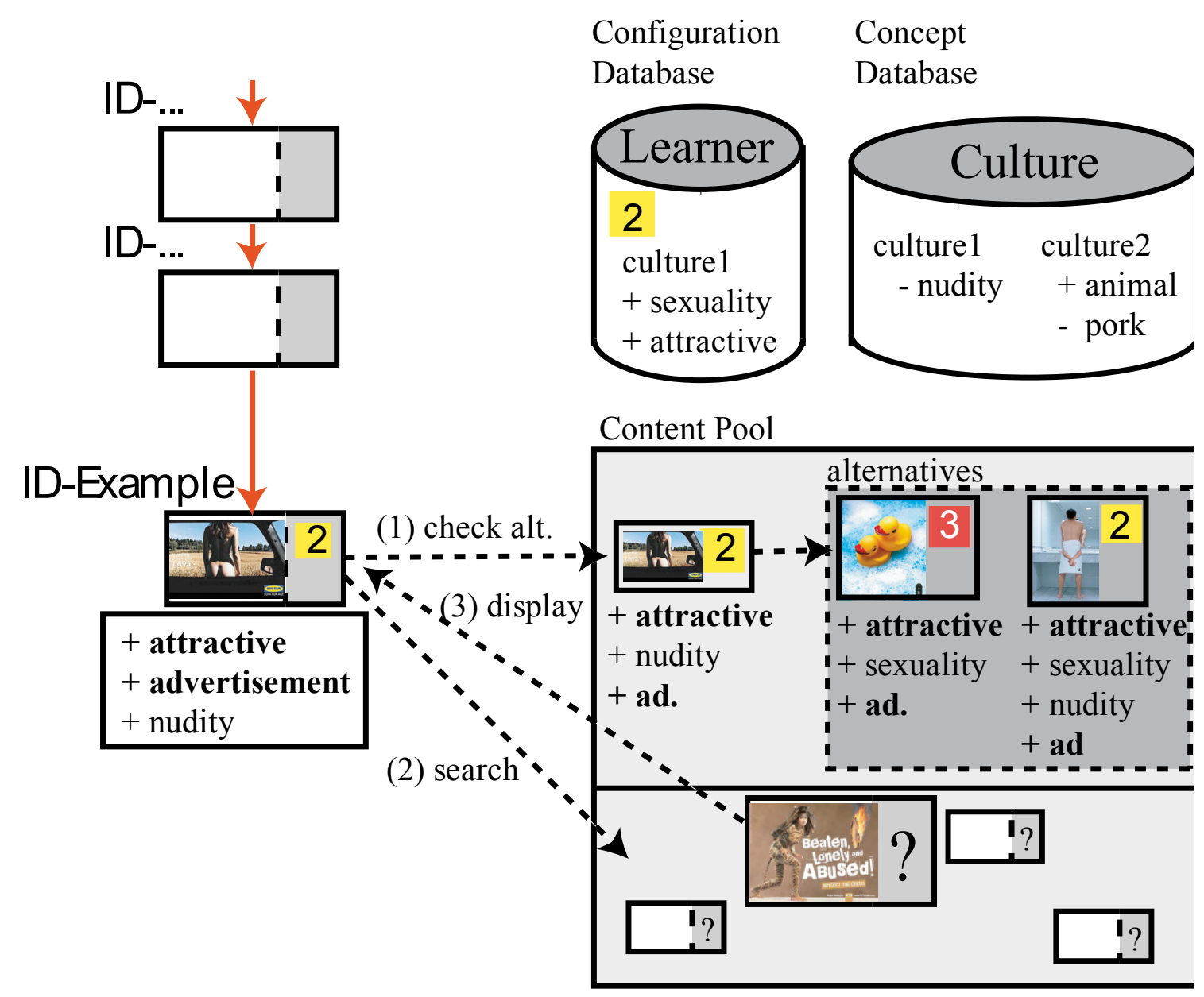

Figure 4: Example for using concepts within the object selection. Based on Reiners \& Sassen (2007)

\section{Conclusion}

We have presented the schema for sophisticated, fine-grained, dynamic, learning path adaptivity set in an e-Learning environment. In the future, these systems will permit cross- and multicultural learning scenarios to be confidently and competently matched with individual user learning needs taking into account, for the first time, cultural sensitivities, respecting the cultural norms and ethics of learners, without the need for special segregation of learners or learning content. Future learners may make university and course selection decisions based on the ability of the educational provider to demonstrably respect and accommodate the diverse cultural norms and ethics found among the peoples of the world. A now well established, but far simpler example, can be found in the air travel industry, where multilingual flight attendants and culturallyspecific meal options are already commonplace.

There are still several questions that are not answered in this contribution. How can it be implemented? Is it working in a real course? Is it accepted by learners and lecturers? Is it used? Even though the research project is not yet finished and many questions cannot be completely answered yet, first indications from a prototype that realized most of the adaptive concept was successfully tested within a virtual classroom setting. We performed several evaluations about acceptance and requirement for the virtual education, indicating a continuation of the work in progress (Sassen, 2007). Nevertheless, the drawback of SmartFrame - our first prototype - was the small number of learning objects, i.e. redundant objects which represent the same content in a different way. This 
is also the main argument for every adaptive approach that relies on the quality of the repository. Inclusion of Internet objects or unknown learning objects was not possible as the quality could not be verified - bear in mind the automatic analysis of the content and verification that the content is correct regarding the current demand. Including the conceptual analysis in our approach, we are able to overcome this problem as we can determine the concepts of an unknown object, on which we can base our selecting process during the search of an alternative.

We are currently implementing several components: (1) an improved repository with dynamic layers representing the various networks of relations between objects, (2) components to edit and visualize objects and relations, (3) improved methods to detect the concepts in an unknown text, and (4) an extended component for the adaptivity and dynamic learning paths. The experiments from SmartFrame and other projects are used to build required components and set up an innovative learning environment, either standalone or integrated in systems like Moodle (Moodle, 2008) or Blackboard (Blackboard, 2008). Note that our overall goal is to improve e-Learning and classroom teaching so that we always keep in mind the people that will later use the system. Therefore, interface development for various user groups is an important topic, next to the necessity of requiring a large content pool and continuous evaluations to verify the progress. Our results will be posted at www.eaglesemantics.com and www.smartframe.de.

\section{Acknowledgements}

The authors would like to acknowledge the Informing Science Institute reviewers for their detailed and helpful comments.

\section{References}

Alavi, M., Maraka, G. M., \& Yoo, Y. (2002). A comparative study of distributed learning environments on learning outcomes. Information Systems Research, 13, 404-415.

Berlanga, A., \& García, F. (2005). Learning technology specifications: Semantic objects for adaptive learning environments. International Journal of Learning Technology, 1(4), 458-472.

Bhattacharya, M., \& Jorgensen, L. (2004). Collaborative research projects in creating distributed learning environment in multicultural context. Third Pan-Commonwealth Forum on Open Learning, Dunedin, New Zealand.

Bhatti, A., Tubaisahat, A., \& El-Qawasmeh, E. (2005). Using technology-mediated learning environment to overcome social and cultural limitations in higher education. In Proceedings of InSITE 2005. Flagstaff, Arizona, USA, June 16-19. Retrieved from http://proceedings.informingscience.org/InSITE2005/I06f77Bhat.pdf

Blackboard. (2008). Blackboard: Educate, Innovate, Everywhere. Retrieved 15/2/2008 from http://www.blackboard.com

Bloomberg. (2003). Toyota kicks off China's first car trademark dispute (Update2). Retrieved 15/2/08 from http://quote.bloomberg.com/apps/news?pid=10000101\&sid=ayOf4GSbbL0I\&refer=japan

Bradsher, K. (2007, April 22). What should a Chinese car look like? The New York Times. Retrieved 15/02/08 from http://www.nytimes.com/2007/04/22/automobiles/22SHOW.html

Collis, B. (1999). Designing for differences: Cultural issues in the design of WWW-based course-support sites. British Journal of Educational Technology, 30(3), 201-217.

Collis, B., \& Remmers, E. (1997). The world wide web in education: Issues related to cross-cultural communication and interaction. In B. Kahn (Ed.), Web-based Instruction (pp. 85-92). New Jersey: Educational Technology Publications. 
Conlan, O., Hockemeyer, C., Wade, V., \& Albert, D. (2002). Meta-data driven approaches to facilitate adaptivity in personalized eLearning systems. Journal of Information and Systems in Education, 1, 3844.

Dreher, H. (2006). Interactive on-line formative evaluation of student assignments. In Proceedings of InSITE 2006, Salford - Greater Manchester, England. June 25-28. Retrieved from http://proceedings.informingscience.org/InSITE2006/IISITDreh235.pdf

Dreher, H., Scerbakov, N., \& Helic, D. (2004). Thematic driven learning. In Proceedings of World Conference on E-Learning in Corporate, Government, Healthcare, and Higher Education 2004, pp. 25942600. Norfolk, VA: AACE.

Dreher, H., \& Williams, R. (2006). Assisted query formulation using Normalised Word Vector and dynamic ontological filtering. In H. Legind Larsen et al. (Eds.), Flexible Query Answering Systems (FQAS 2006),Lecture Notes in Artificial Intelligence (LNAI 4027), pp. 282-294. Springer-Verlag Berlin Heidelberg. Retrieved from http://dx.doi.org/10.1007/11766254 24

Dublin Core. (2008). Dublin Core Metadata Initiative (DCMI). Retrieved 15/2/2008 from http://dublincore.org

Fluck, U. (2008). Multicultural learning solutions: Bibliography. Retrieved 15/2/2008 from http://www.cas.umt.edu/mls/bibliography.htm

Friedman, T. L. (2006). The world is flat: The globalized world in the twenty-first century. London: Penguin Books.

Gaspari, F. (2006). Look who's translating: Impersonations, Chinese whispers and fun with machine translation on the Internet. In EAMT-2006: 11th Annual Conference of the European Association for Machine Translation, June 19-20, Oslo, Norway, pp. 149-158.

Geertz, C. (1973). The interpretation of culture. New York: Basic Books.

Gem Sty. (2007). Top 10 copycat cars. Retrieved 15/2/08 from http://gemssty.com/2006/10/29/top-10copycat-cars

Groeschel, S., \& Doherty, L. (2000). Conceptualising culture. Cross Cultural Management - An International Journal, 7(4), 12-17.

Hampden-Turner, C. M., \& Trompenaars, F. (2000). Building cross-cultural competence. New Haven: Yale University Press.

Hassan-Montero, Y., \& Herrero-Solana, V. (2006). Improving tag-clouds as visual information retrieval interfaces. International Conference on Multidisciplinary Information Sciences and Technologies, InSciT2006.

Jary, D. \& Jary, J. (1991). The HarperCollins dictionary of sociology. New York: Harper Collins.

Larreamendy-Joerns, J., \& Leinhardt, G. (2006). Going the distance with online education. Review of Educational Research, 76(4: Winter), 567-605.

Martin, D. (2007, June 19). Technical update: Risk management in China: Outsourcing and investment. Finance Week. Retrieved 15/2/08 from http://www.financeweek.co.uk/cgibin/item.cgi $\mathrm{id}=5221 \& \mathrm{~d}=302 \& \mathrm{~h}=24 \& \mathrm{f}=254$

Martinez, J. (2008). MPEG-7 overview. Retrieved 15/2/2008 from http://www.chiariglione.org/mpeg/standards/mpeg-7/mpeg-7.htm

McAndrew, P., \& Weller, M. (2005). Applying learning design to supported open learning. In R. Koper \& C. Tattersall (Eds.), Learning design. A handbook on modelling and delivering networked education and training (pp. 281-291). The Netherlands: Springer.

McLoughlin, C. (2001). Inclusivity and alignment: Principles of pedagogy, task and assessment design for effective cross-cultural online learning. Distance Education, 22(1), 7-29. 
Moodle. (2008). Moodle - A free, open source course management system for online learning. Retrieved 15/2/2008 from http://moodle.org

Moynihan, M., Mitchell, S., Pavin, D., Koppitz, R., Wessing, T., Zhang, T., et al. (2004). Intellectual property rights in China: Risk assessment, avoidance strategy and problem solving ('The China IPR guidelines'). The UK China IPR Forum. Retrieved 15/2/08 from http://www.chinabusinesssolutions.com/dbimg/china_ipr_guidelines1.01.pdf

Nilsson, M., Palmer, M., \& Naeve, A. (2002). Semantic web meta-data for e-learning - Some architectural guidelines. Proceedings of the 11th World Wide Web Conference, Hawaii, USA.

Nussbaumer, A., Gütl, C., \& Albert, D. (2007). Towards a web service for competence-based learning and testing. Proceedings of ED-MEDIA 2007. World Conference on Educational Multimedia, Hypermedia \& Telecommunications, 25-29 June, Vancouver BC, Canada, pp. 995-998.

Parhizgar, K. D. (1998). International cross-cultural collaborative teaching and training theories (ICCCTTT). Journal of Teaching in International Business, 9(3), 21-41.

Reiners, T., Reiß, D., \& Sassen, I. (2005). Ontology-based retrieval, authoring, and networking for generalized e-content. In Proceedings of E-Learn 2005: World Conference on E-Learning in Corporate, Government, Healthcare, \& Higher Education, Vancouver BC, Canada, pp. 2349-235.

Reiners, T., Reiß, D., Schulze, H., \& Voß, S. (2003) SmartFrame: An integrated environment for XMLcoded learning material. In W. Uhr, W. Esswein, \& E. Schoop (Eds.), Wirtschaftsinformatik 2003/Band I, Physica, Heidelberg, pp. 613-632.

Reiners, T. \& Sassen, I. (2007). A framework for adaptive learning paths. In T. Kidd \& H. Song (Eds.), Handbook of research on instructional systems \& technology (pp. 117-141). Harrisburg, PA: Idea Group Publishing.

Rogers, P. C., Graham, C. R., \& Mayes, C. T. (2007). Cultural competence and instructional design: Exploration research into the delivery of online instruction cross-culturally. Instructional Technology Forum (ITFORUM). Retrieved 15/2/2008 from http://it.coe.uga.edu/itforum/paper96/Rogers_ITForumPaperHTML.html

Sassen, I. (2007). Virtuelle lehr- und lernumgebungen: Konzeption, didaktisches design und bewertung. [Virtual teaching and learning environments: Concepts, instructional design and evaluation]. Shaker Publishing. Retrieved from www.shaker.eu/Catalogue/details.asp? ID $=8834099 \& C C=655 \& I S B N=3-$ $\underline{8322-6161-3}$

Sassen, I., \& Schwartz-Reinken, B. (2005). Adaptation and configuration: Support-possibilities of different learner types within virtual learning environments - An experience report. In H. Selvaraj \& P. K. Srimani (Eds.), Proceedings of ITCC 2005. International Conference on Information Technology, Las Vegas, Vol. 1 pp. 442-446.

Schüppel, J. (1997). Wissensmanagement: Organisatorisches lernen im spannungsfeld von wissens- und lernbarrieren (1. Aufl.). [Knowledge management: Barriers to learning and knowing in organisational learning contexts (1st ed.)]. Wiesbaden: Deutscher Universitäts-Verlag.

Sims, R., \& Stork, E. (2007). Design for contextual learning: Web-based environments that engage diverse learners. AusWeb: The Thirteenth Australasian World Wide Web Conference. Retrieved 15/2/2008 from http://ausweb.scu.edu.au/aw07/papers/refereed/sims/paper.html

Slay, J. (2002). Human activity systems: A theoretical framework for designing learning for multicultural settings. Educational Technology \& Society, 5(1). Retrieved 15/2/08 from http://www.ifets.info/journals/5 1/slay.html

Stanley, W. B., \& Brickhouse, N. W. (1994). Multiculturalism, universalism and science education. Science Education, 87, 387-398.

Towle, B., \& Halm, M. (2005). Designing adaptive learning environments with learning design. In R. Koper \& C. Tattersall (Eds.), Learning design. A handbook on modelling and delivering networked education and training (pp. 215-226). The Netherlands: Springer. 
UNESCO. (2002). Universal declaration on cultural diversity. Retrieved 15/2/08 from http://www.unesco.org/education/imld_2002/unversal_decla.shtml

Van Rosmalen, P., \& Boticario, J. (2005). Using learning design to support design and runtime adaptation. In R. Koper \& C. Tattersall (Eds.), Learning design: A handbook on modelling and delivering networked education and training (pp. 291-301). The Netherlands: Springer.

Wang, M. (2007). Designing online courses that effectively engage learners from diverse cultural backgrounds. British Journal of Educational Technology, 38(2), 294-311.

Wikipedia. (2007). Concept. Retrieved 15/2/08 from http://en.wikipedia.org/w/index.php?title=Concept\&oldid $=163585052$

Williams, F. (2007). China to foreign automakers: Drop dead. The Truth about Cars. Retrieved 15/2/08 from http://www.thetruthaboutcars.com/china-to-foreign-automakers-drop-dead/

Williams, R. (2006). The power of normalised word vectors for automatically grading essays. In Proceedings of InSITE 2006. Salford - Greater Manchester, England. June 25-28. Retrieved from http://proceedings.informingscience.org/InSITE2006/IISITWill155.pdf

Young, P. A. (2008). Integrating culture in the design of ICTs. British Journal of Educational Technology, 39(1), 6-17.

\section{Biographies}

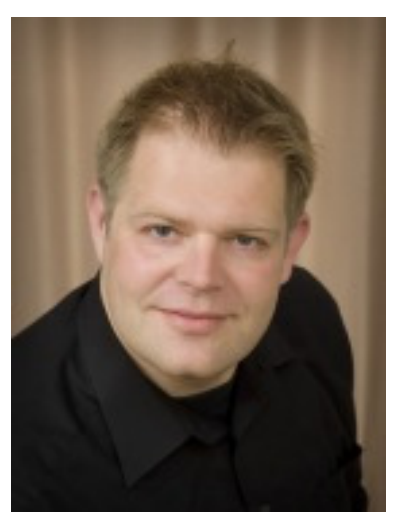

Torsten Reiners is currently a postdoctoral researcher at the University of Hamburg, Germany, and Associate with the Curtin University of Technology in Perth, Australia. His research and teaching experiences are in the areas of clustering and mining large data sets, onlinealgorithms and the incorporation of bio-analogous meta-heuristics in simulations models (applied to container terminals), fleet logistics, information systems as well as several topics in e-learning and software development. Within his PhD-thesis "Simulation and OR with SMARTFRAME" he demonstrated concepts for didactical models. Besides scientific publications, he participated in national and international research projects and is currently doing research in semantic networks to improve cross-border communication and (e)learning as well as machine translation. Another interest is about (virtual) worlds and their interconnectivity and exchange without barriers. This research includes the development of highly adaptive systems, automatic processing of documents and their analysis as well as evaluation, the usage for educational purposes in a multicultural setting, and the usage of innovative platforms like virtual worlds. Torsten Reiners is co-founder of Campus Hamburg and Students@work, an initiative to promote education as well as Hamburg's universities in Web 3D. The co-operation with Heinz Dreher is in the field of conceptual analysis and its application with respect to an automatic assessment lab and adaptive content repository.

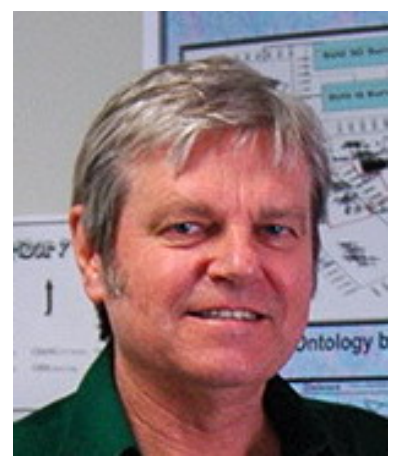

Heinz Dreher is Associate Professor in Information Systems at the Curtin Business School, Curtin University, Perth, Western Australia. He has published in the educational technology and information systems domain through conferences, journals, invited talks and seminars; is currently the holder of Australian National Competitive Grant funding for a 4-year E-Learning project and a 4-year project on Automated Essay Grading technology development, trial usage and evaluation; has received numerous industry grants for investigating hypertext based systems in training and business scenarios; and is an experienced and accomplished teacher, receiving awards for his work in cross-cultural 
awareness and course design. In 2004 he was appointed Adjunct Professor for Computer Science at TU Graz, and continues to collaborate in teaching \& learning and research projects with European partners. Dr Dreher's research and development programme is now supported by Curtin Business School Area of Research Focus funding - Semantic Analysis and Text Mining for Business and Education (www.eaglesemantics.com) in addition to other competitive funding obtained for individual projects. 